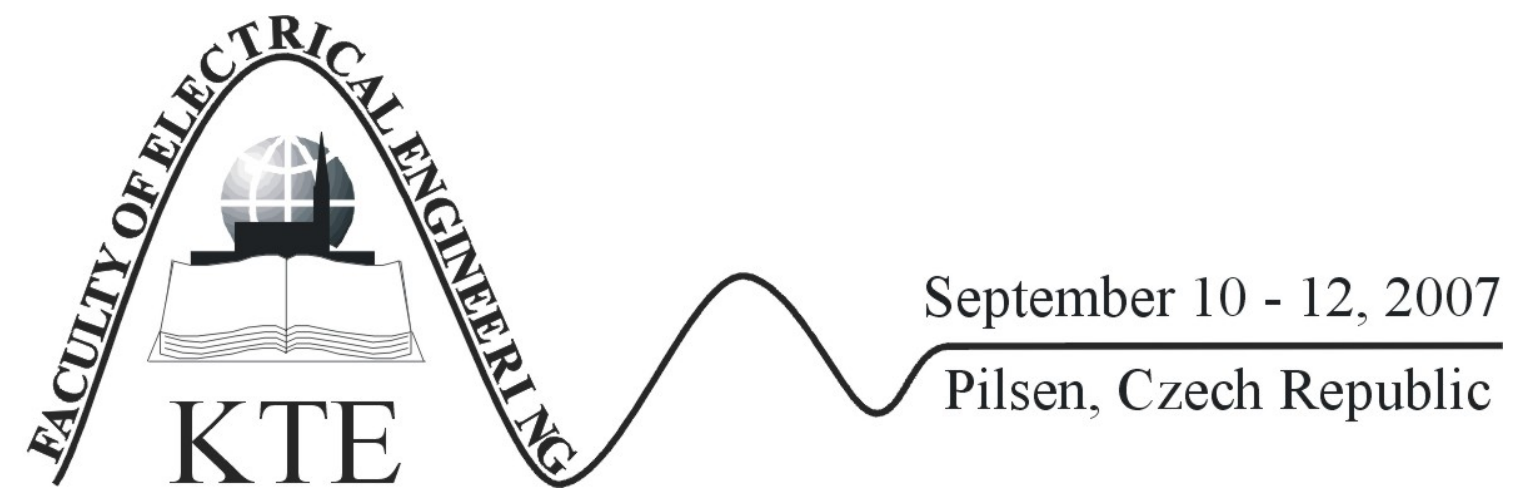

\title{
A SIMPLE NUMERICAL SIMULATION OF INTERNAL STRUCTURE OF PARTICLES TEST
}

\author{
DOC. ING. KAREL BARTUŠEK, DRSC. \\ Doc. Ing. Pavel Fiala, Ph.D. \\ InG. Miloslav SteinbaueR, Ph.D.
}

\begin{abstract}
The article describes a test of numerical model of the electron beam according to present knowledge of references [1][5]. The basic configuration of the electron beam was verified in Institute of Scientific Instruments Academy of Sciences of the Czech Republic experimentally. We prepared the numerical model which is based on the particle theory. Actually, it respects classical Electrodynamics Material Wave Theory (MWT). Numerical results were evaluated. The second model was prepared in respect to theory of wave packet (Louis de Broglie) and solved again. Results of both models were the same in their quality, we evaluated electric field intensity $\boldsymbol{E}$ on the electron impact area, and they corresponded with results from experiments.
\end{abstract}

Key words: material wave theory, wave packet, electron beam, finite element method

\section{INTRODUCTION}

One of the problem, which is solved at the Department of Theoretical and Experimental Electrical Engineering, is the determination of water molecule properties exposed to the electric field. Nuclear magnetic resonance (NMR) is a promising experimental method for this purpose.. The disadvantage of this method is the presence of strong magnetic field. It is not easy to analyze the influence of the electric field because of the above mentioned disadvantage.

The numerical model can serve to solution of this problem. At the first, we can perform the simulation of the magnetic field influence and determine the influence of the electric field consequently. Next, we can make an experiment in the NMR spectrometer and compare experimental results with results of the simulation. This approach allows us to determine the influence of the electric field on the water molecules. When we want to build a suitable numerical model, we have to decide how to interpret the particle theory material theory (MT).

We prepared the numerical models of two known material theory approaches - particle theory (PT) and material wave theory (MWT). Numerical models were verified on a simple example and we have looking for relations among these models. The finite element method (FEM) was used for the model solution. Results obtained by comparison of the PT and MWT models are presented in this paper.

Material wave theory phenomena [1]-[4] can be demonstrated and verified on the simple example, described below. The principles of the material wave theory are presented in [3].

\section{Physical Model}

Numerical models based on the particle theory and material wave theory were built. The test example was built. The example consist of the electrode pair for electron microscope testing [6]. The test example is shown in Fig.1.

In the Fig.1, the electrode system is supplied by high voltage source $U(\mathrm{t})$. Electrons exiting A electrode, are moving toward the $A^{\prime}$ electrode. The A' electrode collects electrons. The electrode system B-B', supplied by the $u_{\mathrm{c}}$ source allows to correct the electron trajectory and the impact point. This test example was solved by the means of finite element method, utilizing ANSYS system and APDL algorithms. The first of them was designed in accordance with the classical macroscopic theory. Results were obtained by the solution of partial differential equations formulated on the basis of reduced Maxwell equations [5]. The solution of the mass point dynamics has utilized the coupled electrostatic model and the Lorentz force law: 


$$
\boldsymbol{F}=q \boldsymbol{E}+q \boldsymbol{v} \times \boldsymbol{B} \text { in } \Omega,
$$

where $\mathrm{F}$ is the vector of the resultant force, which acts on the particle, $\mathrm{q}$ is the particle charge, $\mathrm{E}$ is the external electric field intensity vector, $\mathrm{v}$ is the speed of the moving particle, $B$ is the magnetic flux density vector of the external magnetic field, $\Omega$ is the definition area of the model. Obtained results were experimentally verified on the Institute of Scientific Instruments of the Academy of Sciences of the Czech Republic.

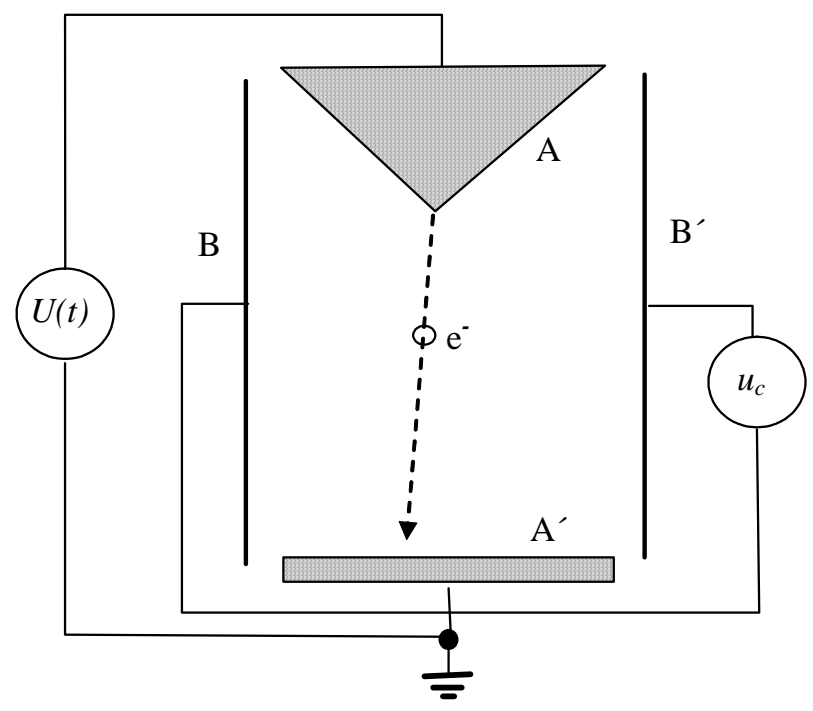

Fig.1. The example setup for the MWT test

Fig.3 shows the result of the numerical model of the electrode system test from Fig.1. Simulation results correspond with results obtained by the experiments of the Institute of Scientific Instruments. The parameters of the experiment were following: $U(\mathrm{t})=U_{\max } \cdot f(\mathrm{t})$, where $U_{\max }=10 \mathrm{kV}, u_{\mathrm{c}}=10 \mathrm{~V}$, the distance of the B-B' electrode $d_{\mathrm{BB}},=20 \mathrm{~mm}$, the distance of the A-A' electrode $d_{\mathrm{AA}}$, $=50 \mathrm{~mm}$. We can evaluate the count of electrons, collected by the A' electrode, as the space charge $q_{\mathrm{d}}$ with spatial distribution

$$
\boldsymbol{E}=\frac{1}{4 \pi \varepsilon_{S}} \int_{|\boldsymbol{r}|^{2}} \frac{\sigma}{\boldsymbol{S}} \text { in } \Gamma_{\mathrm{A}^{\prime}},
$$

where $\varepsilon$ is the vacuum permittivity, $\sigma$ is the surface charge density, $\boldsymbol{r}$ is the position vector, $\boldsymbol{S}$ is the oriented surface. After the evaluation (Fig.4), Fig.5 shows the module of the electric field intensity vector on the $\Gamma_{A^{\prime}}$. boundary. Results of the particle theory model were compared with results of the MWT model. The MWT model utilizes the wave equation. The wave equation is formulated with the help of Maxwell equations in the partial differential form [5]. Boundary conditions were obtained by the PT model solution.
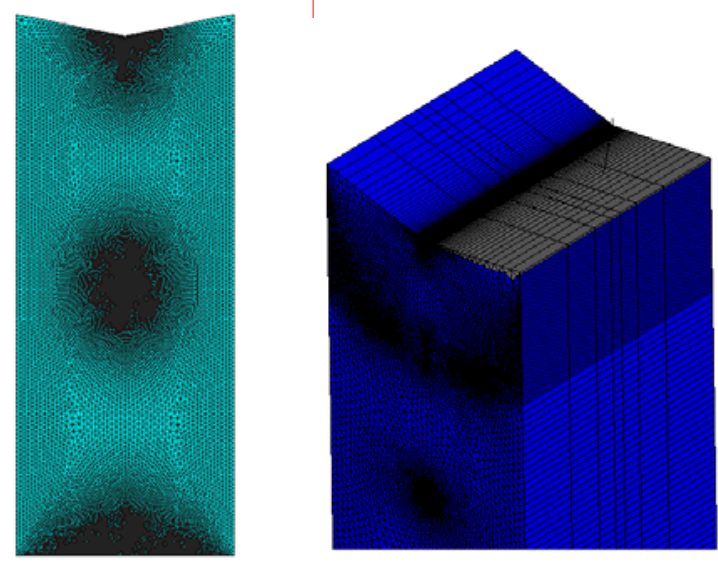

Fig.2. The FEM mesh for moving particle model.

Boundary conditions are represented by intensities of the electric field on the electrode system surface in Fig.3. Here, $\quad E_{\max 1}=670 \mathrm{~V} / \mathrm{m}, \quad E_{\max 2}=2,9.10^{5} \mathrm{~V} / \mathrm{m}$, $E_{\max 3}=2,1 \cdot 10^{7} \mathrm{~V} / \mathrm{m}$.
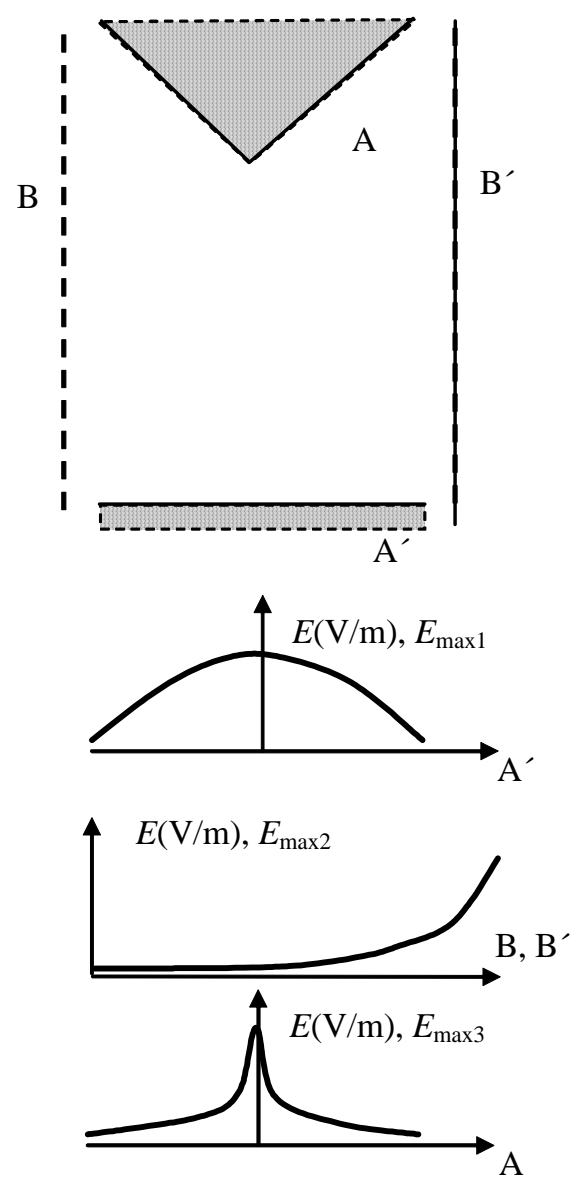

Fig.3. Boundary conditions obtained by the solution of the PT model. 
The model of the source of the electromagnetic field was simplified:

$$
\boldsymbol{E}(t)=\boldsymbol{E}(x, y, z) \cdot \sin g(t)) \text { in } \Gamma_{\mathrm{A}},
$$

where $\boldsymbol{E}(\mathrm{x}, \mathrm{y}, \mathrm{z})$ is the vector of the maximal electric field intensity on the A electrode, $\Gamma_{\mathrm{A}}$ is the surface of the electrode, $\boldsymbol{E}(\mathrm{x}, \mathrm{y}, \mathrm{z})=\boldsymbol{E}_{\text {max. }} \cdot \mathrm{h}(\mathrm{x}, \mathrm{y}, \mathrm{z}), \boldsymbol{E}_{\max }=670 \mathrm{~V} / \mathrm{m}, \boldsymbol{g}(\mathrm{t})$ is the exciting function on the surface $\mathrm{A}$, for simplicity $g(\mathrm{t})=\sin (2 \pi f)$ and $f=3.10^{10} \mathrm{~Hz}$. This simplification was considered, because of the reasonable computing time. In accordance with the MWT the appropriate electron frequency should be $f=3 \cdot 10^{27} \mathrm{~Hz}$

$$
f=\frac{c}{\lambda}
$$

where $c$ is the speed of light, $\lambda$ represents the electron size $\left(\lambda=1 \cdot 10^{-19} \mathrm{~m}\right)$. The solution with valid electron size is currently verified.

\section{Discussion}

Performed experiments show, that it is possible to substitute the macroscopic, microscopic material model (described with the particle theory) with the material wave theory. The powerful tool for basic material models was discovered [1]-[4]. It is possible to explain many of experiments with the help of this new approach.
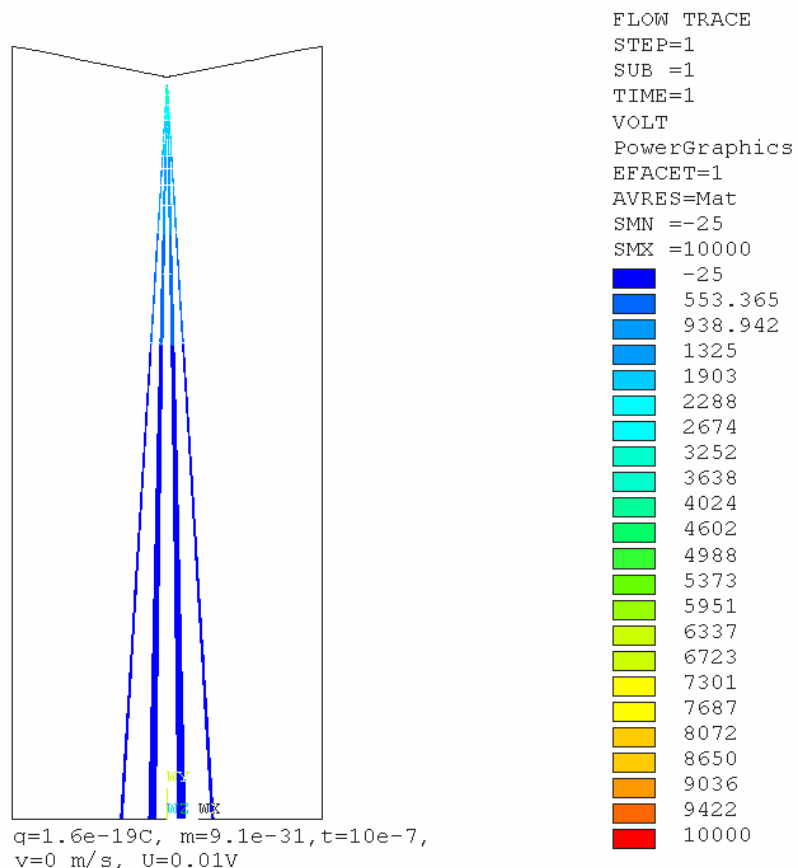

Fig.4. The resultant trajectory of the moving electrons in the electrode system - particle theory model.

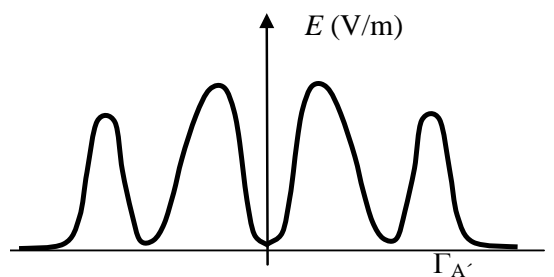

Fig.5. The electric field intensity E module - particle theory model.

\section{Conclusion}

On the basis of the experiments mentioned above, the model of basic particles will be built. The next point is to set up the model of basic elements. This activity is connected with the research plan FRVS CR No. MSM 0021630513 ELCOM, No. MSM 0021630516. The aim of this research is to describe the water molecule behaviour in the electric field, with know intensity $E$. The behaviour is experimntaly tested $n$ the NMR spectrometer.

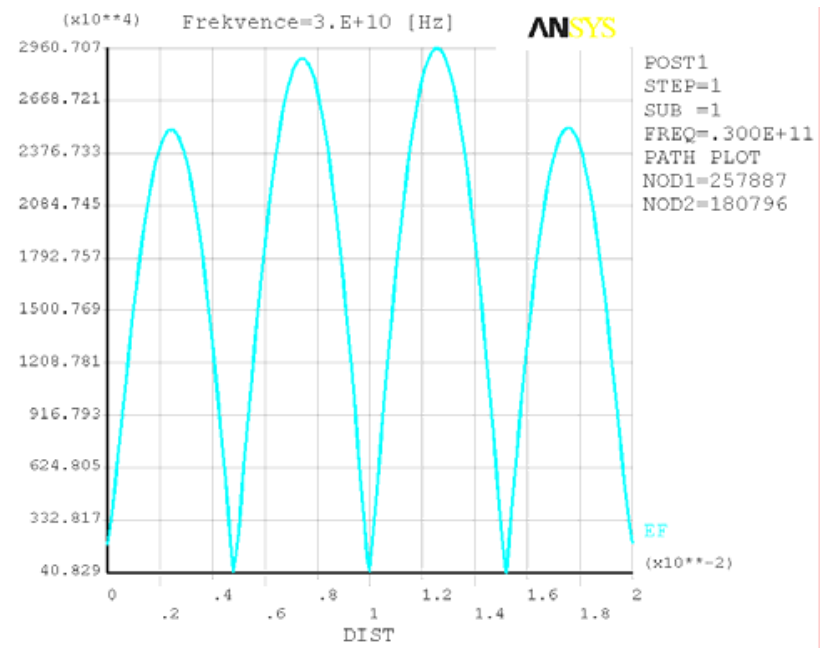

Fig.6. The electric field intensity E module - MWT model.

\section{ACKNOWLIDGEMENT}

The research described in the paper were financially supported by research plans GAAV B208130603, MSM 0021630516, GA102/07/0389, KJB208130603 and MSM0021630513. 


\section{REFERECES}

[1] van Vlaenderen K. J. and Waser A.:Electrodynamics with the scalar field. Physics, Vol.2, 1-13, 2001.

[2] Kikuchir H.: Electrohydrodynamics in dusty and dirty plasmas, gravito-electrodynamics and EHD. Kluwer academic publishers, Dordrecht/Boston/London, 2001.

[3] van Vlaenderen K. J.: A charge space as the orogin of sources, fields and potentials. Physics, arXiv: physics/9910022 v1 16 Oct 1999, 1-13, 1999.

[4] Hofer W. A.: A charge space as the origin of sources, fields and potentials. Physics, arXiv: quant-ph/ 9611009 v3 17 Apr 1997, 1-13, 1997.

[5] Prosser V.: Experimentální metody biofyziky. Academia, Praha, 1989.

[6] Delong A.: Verbal information. Czech Academy of Science, ISI Brno, 7.2.2006.

Pavel Fiala

Karel Bartušek

Miloslav Steinbauer

Department of Theoretical and Experimental Electrical Engineering

Brno University of Technology

Kolejni 2906/4

61200 Brno, Czech Republic

E-mail: fialap@feec.vutbr.cz bartusek@feec.vutbr.cz steinbau@feec.vutbr.cz 